\title{
Effect of Antiferromagnetic Interlayer Coupling on Current-Assisted Magnetization Switching
}

\author{
S. Urazhdin, H. Kurt, W. P. Pratt Jr., and J. Bass \\ Department of Physics and Astronomy, Center for Fundamental Materials Research, \\ and Center for Sensor Materials, Michigan State University, East Lansing, MI 48824-2320
}

\begin{abstract}
We compare magnetization switching in $\mathrm{Co} / \mathrm{Cu} / \mathrm{Co}$ nanopillars with uncoupled and dipole-field coupled Co layers. In uncoupled nanopillars, current-driven switching is hysteretic at low magnetic field $\mathrm{H}$ and changes to reversible, characterized by telegraph noise, at high $\mathrm{H}$. We show that dipolar coupling both affects the switching current and causes the switching to become reversible at small $\mathrm{H}$. The coupling thus changes the switching to reversible, hysteretic, and then reversible again as $\mathrm{H}$ increases. We describe our results in terms of current-assisted thermal activation.
\end{abstract}

PACS numbers: 73.40.-c, 75.60.Jk, 75.70.Cn

Observations of current-driven magnetization direction switching in $\mathrm{Co} / \mathrm{Cu} / \mathrm{Co}$ [1, 2, 3, 4, 4, 5, 6, 7] and $\mathrm{Py} / \mathrm{Cu} / \mathrm{Py}$ $\left[\mathrm{Py}=\right.$ Permalloy $\left.=\mathrm{Ni}_{84} \mathrm{Fe}_{16}\right]$ [8] nanopillars have generated great interest, both for science - studies of magnetic systems driven far out of equilibrium, and technologyin magnetic random access memory (MRAM) this effect might eliminate the need for magnetic field-driven switching. In this Letter we show that dipolar coupling between the magnetic layers can affect the switching. Specifically, we show that the switching current is not determined solely by the switching mechanism [3, 5], but varies with dipolar coupling, and that sufficiently strong antiferromagnetic (AF) coupling leads to reversible (nonhysteretic) switching at small magnetic field $\mathrm{H}$. This effect may find application in high-sensitivity field sensors, e.g. read-heads of computer hard drives.

Our devices were nanofabricated using the following steps. First, a $\mathrm{Cu}(80) / \mathrm{Co}(20) / \mathrm{Cu}(6-$ $10) / \mathrm{Co}(2.5) / \mathrm{Cu}(5) / \mathrm{Au}(15)$ multilayer was sputtered onto an oxidized Si wafer in Ar pressure of 2 mTorr. All thicknesses in this Letter are in nanometers. $\mathrm{An} \mathrm{Al}(50)$ nanopillar with lateral dimensions of about $70 \mathrm{~nm}$ by $130 \mathrm{~nm}$ was then formed by a combination of e-beam lithography and evaporation. The $\mathrm{Al}$ was used as a mask for ion-milling the multilayer into a nanopillar. Dipolar coupling between the two Co layers was minimized by timing the ion-milling to stop in the $\mathrm{Cu}$ layer above the bottom $\mathrm{Co}(20)$ layer. When desired, AF dipolar coupling was achieved by continuing the ion-milling about half way through the bottom $\mathrm{Co}(20)$ layer. Magnetic poles at the edges of the two patterned Co layers then AF-couple to minimize the magnetic energy. Without breaking the vacuum, a $\mathrm{SiO}(25-40)$ layer was deposited for electrical insulation between the device electrodes. The $\mathrm{Al}$ mask was removed by ion-milling with the ion-beam parallel to the sample surface, followed by wet etching. The ion-milling removed metals back-sputtered onto the $\mathrm{Al}$ mask. Finally, after a short ion-milling to clean the surface, a $\mathrm{Au}(150)$ top contact was sputtered onto the top Au layer. All measurements were performed at room temperature $(295 \mathrm{~K})$. Differential resistances dV/dI were measured using a standard four-probe setup with lock-in
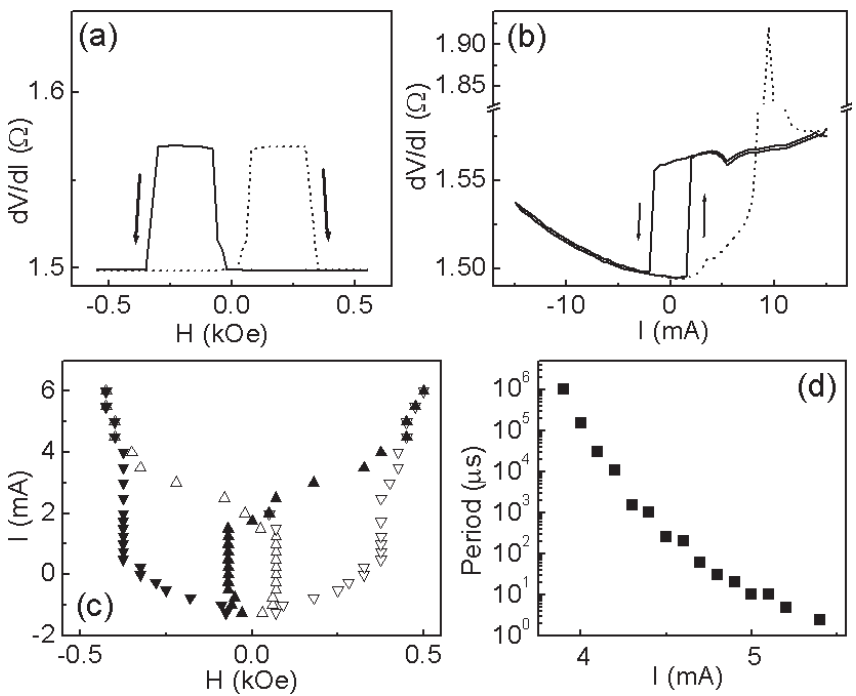

FIG. 1: Magnetization switching in an uncoupled sample. (a) H-dependence of $\mathrm{dV} / \mathrm{dI}$ at $\mathrm{I}=0$. (b) Current dependence of $\mathrm{dV} / \mathrm{dI}$ : Solid line: $\mathrm{H}=0$ Oe, dashed line: $\mathrm{H}=600$ Oe. Arrows mark the scan direction. (c) Switching diagram extracted from the $\mathrm{H}$ scans at various fixed values of I. Upward triangles: $\mathrm{P} \rightarrow \mathrm{AP}$ switching, as defined in the text. Downward triangles: $\mathrm{AP} \rightarrow \mathrm{P}$ switching. Open symbols: $\mathrm{H}$ scanned from negative to positive, closed symbols: reverse H-scan. (d) Variation of the average telegraph noise period with $\mathrm{I}$, as $\mathrm{H}$ is varied between 426 Oe and 479 Oe to keep $\tau_{P}=\tau_{A P}$.

detection, adding an ac current of amplitude $20 \mu \mathrm{A}$ at $8 \mathrm{kHz}$ to the dc current I. Most uncoupled devices tested had room temperature resistances of about $1.5 \Omega$ and magnetoresistances (MR) of about $5 \%$, similar to the best MR values reported by others 1]. The coupled devices had larger resistances (because of the additional interface and Co layer in the nanopillar) but similar MRs. We define positive current to flow from the bottom (extended) to the top Co layer.

Typical data for an uncoupled sample are shown in Fig. 11 and comparative data for a coupled sample are shown in Fig. 2] We begin with the uncoupled sample, the data for which are consistent with prior studies of 
uncoupled $\mathrm{Co} / \mathrm{Cu} / \mathrm{Co}$ [2] and $\mathrm{Py} / \mathrm{Cu} / \mathrm{Py}$ [8]. Fig. 1(a) shows that the MR at $\mathrm{I}=0$ is symmetrically hysteretic in $\mathrm{H}$ and the increase in resistance from the low resistance parallel $(\mathrm{P})$ orientation of magnetic moments to the high resistance anti-parallel (AP) orientation occurs in a single sharp step. The current-driven switching is hysteretic at $\mathrm{H}=0$ Oe (solid curve in Fig. 1(b)), with switching to the high resistance anti-parallel (AP) state at positive $I_{s}^{P \rightarrow A P}$, and to the low resistance parallel $(\mathrm{P})$ state at negative $I_{s}^{A P \rightarrow P}$. The resistances and their changes in Fig. 1(b) are close to those in Fig. 1(a). The dotted curve in Fig. I(b) shows that at large enough $\mathrm{H}$ the hysteretic step in $\mathrm{dV} / \mathrm{dI}$ turns into a non-hysteretic (reversible) switching peak.

Fig. 1(c) shows the switching diagram obtained from MR data such as those in Fig. 1(a). We attribute the slight $\mathrm{H}$-asymmetry of the diagram to a combination of the current-induced Oersted field and sample shape asymmetry. The $\mathrm{P} \rightarrow \mathrm{AP}$ switching field $H_{s}^{P \rightarrow A P} \approx 70 \mathrm{Oe}$ is independent of I over the range $-1.7 \mathrm{~mA}<I<1.7 \mathrm{~mA}$. We attribute the $\mathrm{P} \rightarrow \mathrm{AP}$ switching in this range to reversal of the extended Co layer, unaffected by the small current density in that layer. We associate the $\mathrm{AP} \rightarrow \mathrm{P}$ transition with reversal of the thin patterned Co layer at $H_{s}^{A P \rightarrow P}$ determined by its shape anisotropy. At $I>1.7 \mathrm{~mA}, H_{s}^{P \rightarrow A P}$ strongly varies with I. In this regime, we attribute the $\mathrm{P} \rightarrow \mathrm{AP}$ transition to reversal of the patterned Co layer, induced by $I>0$. As the extended Co layer reverses at $H \approx 70$ Oe, the patterned Co layer reverses simultaneously to keep the AP configuration favored by $I>0$. Such simultaneous reversal sometimes produces a weak feature in $\mathrm{dV} / \mathrm{dI}$ at small $\mathrm{H}$.

At $I>4 \mathrm{~mA}$, the data in Fig. 1(c) become nonhysteretic. Time resolved measurements show that the non-hysteretic switching peak in $\mathrm{dV} / \mathrm{dI}$ is characterized by telegraph noise switching between the $\mathrm{P}$ and $\mathrm{AP}$ states, with random distribution of dwell times in the $\mathrm{P}$ state $\left(\tau_{P}\right)$ and AP state $\left(\tau_{A P}\right)$ [8]. When I is increased, and $\mathrm{H}$ adjusted to keep $\tau_{P}=\tau_{A P}$, the average noise period decreases approximately exponentially (Fig. \(d)). The switching diagram, Fig. 1(c), is asymmetric with respect to the current direction; switching is hysteretic at $I<0$, and non-hysteretic only at large $I>0$. This difference occurs because application of $\mathrm{H}$ favors the $\mathrm{P}$ state, while $I>0$ favors the AP state.

Fig. 2 shows data similar to those in Fig. 11 but for a sample with strong AF dipolar coupling between the two Co layers, produced by partial patterning of the extended $\mathrm{Co}(20)$ layer. Again, the field-driven MR (Fig. 2(a)) is hysteretic and approximately symmetrically about $H=0$. But now the MR contains multiple steps, likely because the highly inhomogeneous dipole field favors nonuniform magnetization states of the nanopillar. We determine the switching points from the jump into or from the lowest resistance state. As $\mathrm{H}$ is reduced from a large positive value (solid line in Fig. 2(a)), coupling between the two Co layers causes the thinner Co (2.5) layer to switch to the AP state at $H=0.16 \mathrm{kOe}$, then
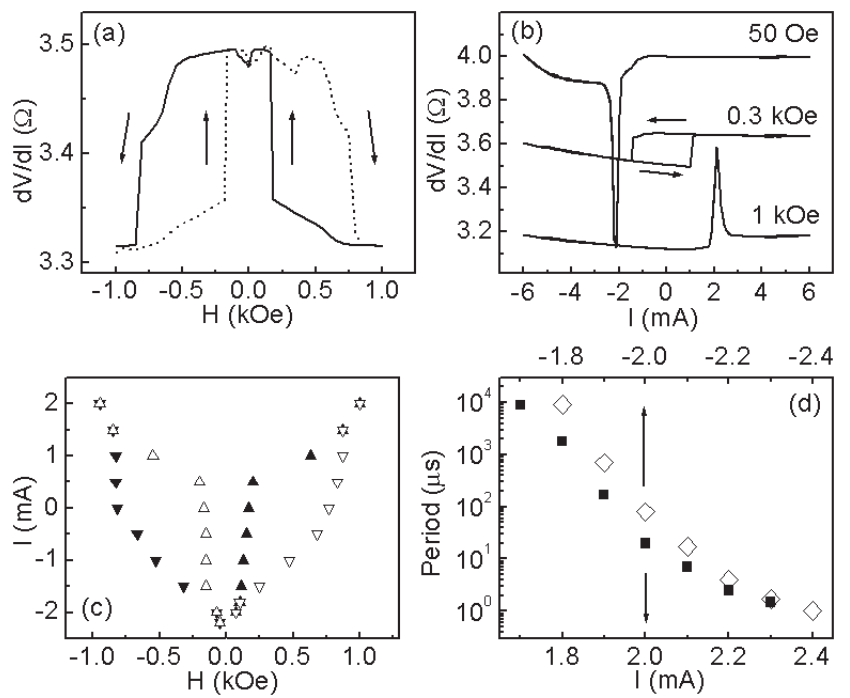

FIG. 2: Magnetization switching in a dipole-coupled sample. (a) $\mathrm{H}$ dependence of $\mathrm{dV} / \mathrm{dI}$ at $\mathrm{I}=0$. (b) Current dependence of $\mathrm{dV} / \mathrm{dI}$ at various $\mathrm{H}$. Curves are offset for clarity. Arrows mark the scan direction at $\mathrm{H}=0.3 \mathrm{kOe}$. (c) Switching diagram extracted from the $\mathrm{H}$ scans at various fixed values of $\mathrm{I}$. Upward triangles: $\mathrm{P} \rightarrow \mathrm{AP}$ switching, as defined in the text. Downward triangles: $\mathrm{AP} \rightarrow \mathrm{P}$ switching. Open symbols: $\mathrm{H}$ scanned from negative to positive, closed symbols: reverse $\mathrm{H}$ scan. (d) Variation of the average telegraph noise period with $\mathrm{I}$, as $\mathrm{H}$ is varied to keep $\tau_{P}=\tau_{A P}$. Solid symbols: $I>0$ (bottom scale), $\mathrm{H}$ varied between 854 and 968 Oe. Open symbols: $I<0$ (top scale), $\mathrm{H}$ varied between 57 and 128 Oe.

at small $H<0$ both the thicker and thinner Co layers flip together to stay in the AP state, and finally at $H \approx-0.8 \mathrm{kOe}$ the $\mathrm{Co}(2.5)$ layer reverses again to return to the $\mathrm{P}$ state.

At small $\mathrm{H}=50$ Oe the behavior as a function of $\mathrm{I}$ in Fig. 2(b) is quite different from that in Fig. 1(b); instead of hysteretic switching, the data show a non-hysteretic, downward peak. This behavior is the most important feature of the data for coupled samples. In Fig. 2(b), the switching is non-hysteretic for small $\mathrm{H}$, becomes hysteretic for intermediate $\mathrm{H}=0.3 \mathrm{kOe}$, and then nonhysteretic again for large enough H. Fig. 22 (c) shows the switching diagram. In contrast to Fig. I(c), all switching points now represent reversal of the the $\mathrm{Co}(2.5)$ layer and switching becomes reversible at large enough magnitude of current in both directions, at small $\mathrm{H}$ for negative I, and large $\mathrm{H}$ for positive I. The symmetry between effects of positive and negative current is extended further by time-resolved measurements of telegraph noise, which is present close to the reversible switching points for both current directions. Fig. 2(d) shows that as the magnitude of $\mathrm{I}$ is increased, while adjusting $\mathrm{H}$ to keep $\tau_{P}=\tau_{A P}$, the telegraph noise period decreases approximately exponentially at similar rates for both current directions. Not all samples with partially patterned bottom Co layer exhibited non-hysteretic switching at small H. Due to weaker AF-coupling, some samples exhibited 


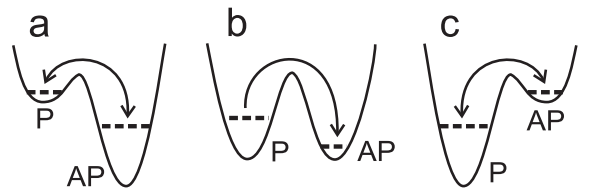

FIG. 3: (a) Schematic of current-driven telegraph noise in AF-coupled sample at $I<0$, small $\mathrm{H}$. Dashed lines indicate $T_{m}$. (b) Same as (a), at $I>0, \mathrm{H}$ close to the dipole field. (c) Same as (a), at $I>0$, large $\mathrm{H}$.

only a dip in $I_{s}^{P \rightarrow A P}$ at small $\mathrm{H}$, resulting in switching diagrams intermediate between Fig. 1(c) and Fig. 2(c).

We interpret the data in Figs. 112 in terms of thermal activation over an effective switching barrier [4, 6, 8]. In $\mathrm{Co} / \mathrm{Cu} / \mathrm{Co}$ nanopillars, both the semi-classical spintorque [9] and quantum threshold [10, 11] models predict magnetic excitation in the $\mathrm{P}$ state at $I>0$ and in the AP state at $I<0$. Such current-driven excitation can be described in terms of a current-dependent effective switching barrier [12], or a current-dependent effective magnetic temperature $T_{m}$, with an effective barrier modified by the current only through the variation of magnetization with temperature [6, 8]. Here $T_{m}$ depends on the magnetic configuration, $T_{m}^{A P}(I) \neq T_{m}^{P}(I)$. These alternative approaches give mathematically similar results, but differ in details that still need experimental testing. We use the latter model, which we find more transparent.

In magnetically uncoupled samples, the magnetization orientation of the patterned layer is bistable at $H=0$, $I=0$ : at $\mathrm{T}=295 \mathrm{~K}$, the barrier height for switching between the two magnetization orientations significantly exceeds the thermal energy. In AF coupled samples at small $\mathrm{H}$, the $\mathrm{P} \rightarrow \mathrm{AP}$ switching barrier is reduced by the dipolar field, leading to a thermally activated $\mathrm{P} \rightarrow \mathrm{AP}$ transition. The reverse $\mathrm{AP} \rightarrow \mathrm{P}$ transition cannot be thermally activated because the corresponding switching barrier is significantly higher. Thus, at $\mathrm{I}=0, \mathrm{H}=0$, $\mathrm{AP}$ is the only stable orientation of the nanopillar, as seen in Fig. 2(b,c). Current-driven magnetic excitation at $I<0$ increases $T_{m}^{A P}$. At sufficiently high $I<0$ the $\mathrm{AP} \rightarrow \mathrm{P}$ transition becomes thermally activated, leading to telegraph noise switching between the AP and $\mathrm{P}$ states (Fig. [3(a)). At larger $\mathrm{H}=0.3 \mathrm{kOe}$ in Fig. 2(b), $\mathrm{H}$ nearly compensates the dipole field, leading to hysteretic switching similar to that in Fig. I(a) at $\mathrm{H}=0$ in an uncoupled sample, and illustrated in Fig. 3(b). As $\mathrm{H}$ is further increased, both the uncoupled and coupled samples behave similarly; As shown in Fig. B(c), the $\mathrm{AP} \rightarrow \mathrm{P}$ transition becomes thermally activated. At large enough $I>0$ the $\mathrm{P} \rightarrow \mathrm{AP}$ transition also becomes activated due to current-induced increase of $T_{m}^{P}$, leading to telegraph noise both in uncoupled (Fig. I(d)) and AFcoupled (Fig. 2(d)) nanopillars.

In summary, we have shown that dipolar AF coupling between magnetic layers leads to reversible currentdriven magnetization switching at small H. Similarly, reversible switching at small $\mathrm{H}$ should be induced by the opposite current direction in ferromagnetically exchanged-coupled samples. We will demonstrate such behavior elsewhere [13]. Thus, the switching current and hysteresis are not intrinsic characteristics of the currentdriven switching mechanisms, as they are strongly affected by the coupling between the magnetic layers.

Fig. 2( (c) shows that, in the reversible switching regime at small $\mathrm{H}$, the switching field of AF-coupled magnetically nanopillars can be adjusted by changing the applied current. In this regime, magnetically coupled nanopillars with exchange-biased extended magnetic layer and zeroed-out switching field may find application as high sensitivity field sensors.

We acknowledge helpful discussions with Norman O. Birge, important contributions to the development of the sample preparation technique by K. Eid and J. Caballero, and support from the MSU CFMR, CSM, the MSU Keck Microfabrication facility, the NSF through Grants DMR 02-02476 and 98-09688, and Seagate Technology.
[1] J.A. Katine, F.J. Albert, R.A. Buhrman, E.B. Myers and D.C. Ralph, Phys. Rev. Lett. 84, 3149 (2000).

[2] F.J. Albert, J.A. Katine, R.A. Buhrman, and D.C. Ralph, Appl. Phys. Lett. 77, 3809 (2000).

[3] J. Grollier, V. Cros, A. Hamzic, J.M. George, H. Jaffres, A. Fert, G. Faini, J.B. Youssef, and H. Legall, Appl. Phys. Lett. 78, 3663 (2001); ibid, Phys. Rev. B (in press).

[4] E. B. Myers, F.J. Albert, J.C. Sankey, E. Bonet, R.A. Buhrman, and D.C. Ralph, Phys. Rev. Lett. 89, 196801 (2002).

[5] F. J. Albert, N.C. Emley, E.B. Myers, D.C. Ralph, and R.A. Buhrman, Phys. Rev. Lett. 89, 226802 (2002).

[6] J. E. Wegrowe, H. Hoffer, Ph. Guittienne, A. Fabian, L. Gravier, T. Wade, and J. P. Ansermet, J. Appl. Phys. 91, 6806 (2002).
[7] J. Z. Sun, D.J. Monsma, T.S. Kuan, M.J. Rooks, D.W. Abraham, B. Oezyilmaz, A.D. Kent, and R.H. Koch, J. Appl. Phys. (in press).

[8] S. Urazhdin, N. O. Birge, W.P. Pratt Jr., and J. Bass, cond-mat/0303149

[9] J. Slonczewski, J. Magn. Magn. Mater. L1, 159 (1996).

[10] L. Berger, Phys. Rev. B 54, 9353 (1996).

[11] M. Tsoi, A.G.M. Jansen, J. Bass, W.C. Chiang, M. Seck, V. Tsoi, and P. Wyder, Phys. Rev. Lett. 80, 4281 (1998); 81, 493(E) (1998).

[12] S. Zhang, cond-mat/0302339

[13] S. Urazhdin, N.O. Birge, W.P. Pratt Jr., J. Bass, to be published. 